\title{
Controlled Growth and Spectroscopy Study of Oxide Nanobelts
}

\author{
Zhong Lin (ZL) Wang * and Yong Ding
}

School of Material Science and Engineering, Georgia Institute of Technology

Atlanta GA 30332-0245, USA

* E-mail: zhong.wang@mse.gatech.edu

One-dimensional (1D) nanostructures (nanowires, nanobelts and nanorods) are the forefront nanomateirals for nanotechnology. Oxide nanostructures have been synthesized for a wide range of semiconducting oxides [1] that are potential building blocks for constructing numerous nanodevices. Using the technique demonstrated for measuring the mechanical properties of nanotubes $[2,3]$, the mechanical and field emission properties of the oxide nanobelts have been characterized. Field effect transistors [4], ultra-sensitive nano-size gas sensors [5], nanoresonators and nanocantilevers [6] have been fabricated using nanobelts.

Structurally, the wurtzite-structured $\mathrm{ZnO}$ is non-central symmetric, and its property is anisotropic as well. It will be very interesting to investigate the electronic structure of $\mathrm{ZnO}$ nanostructures of different growth orientation. However, due to the diversity and lower purity of the samples prohibit the application of the well-established techniques, such as XPS, AES and UPS. Electron energy-loss spectroscopy (EELS) has a high spatial resolution and the possibility of investigating the electronic structure of a nanobelt and a nanotube. By combining the crystallographic and chemical information provided by transmission electron microscopy, EELS may have certain advantages for investigating nano scale electronic structure although its energy resolution is limited.

Electron energy-loss spectroscopy has been used to study the electronic structure of $\mathrm{ZnO}$ nanobelts [7]. The polar surface dominant [01 $\overline{1}$ 0] growth $\mathrm{ZnO}$ nanobelts show an extra surface plasmon peak at 13.0 $\mathrm{eV}$ compared to that from the non-polar surface dominant $[2 \overline{1} \overline{1} 0]$ growth nanobelts, which is suggested to be related to the polar surface. Crystallographic orientation dependence on the energy-loss near-edge structure of different types of nanobelts has been investigated. The observed fine structures at the $\mathrm{O} \mathrm{K}-$ edge and Zinc $\mathrm{L}_{3}$ edge agree with the calculated results.

\section{References:}

[1] Z.W. Pan, Z.R. Dai and Z.L. Wang, Science, 209 (2001) 1947.

[2] P. Poncharal, Z.L. Wang, D. Ugarte and W.A. de Heer, Science, 283 (1999) 1513.

[3] R.P. Gao, Z.L. Wang, Z.G. Bai, W. de Heer, L. Dai and M. Gao, Phys. Rev. Letts., 85 (2000) 622.

[4] M. Arnold, P. Avouris, Z.L. Wang,. Phys. Chem. B, 107 (2002) 659.

[5] E. Comini, G. Faglia, G. Sberveglieri, Zhengwei Pan, Z. L. Wang Applied Physics Letters, 81 (2002) 1869.

[6] W. Hughes and Z.L. Wang, Appl. Phys. Letts., 82 (2003) 2886.

[7] Y. Ding, Z.L. Wang, J. Electron Microscopy, 54 (2005) 287.

[8] Thanks the support from NSF, DARPA, NASA and Airforce.

[9] for details see: http://www.nanoscience.gatech.edu/zlwang/ 

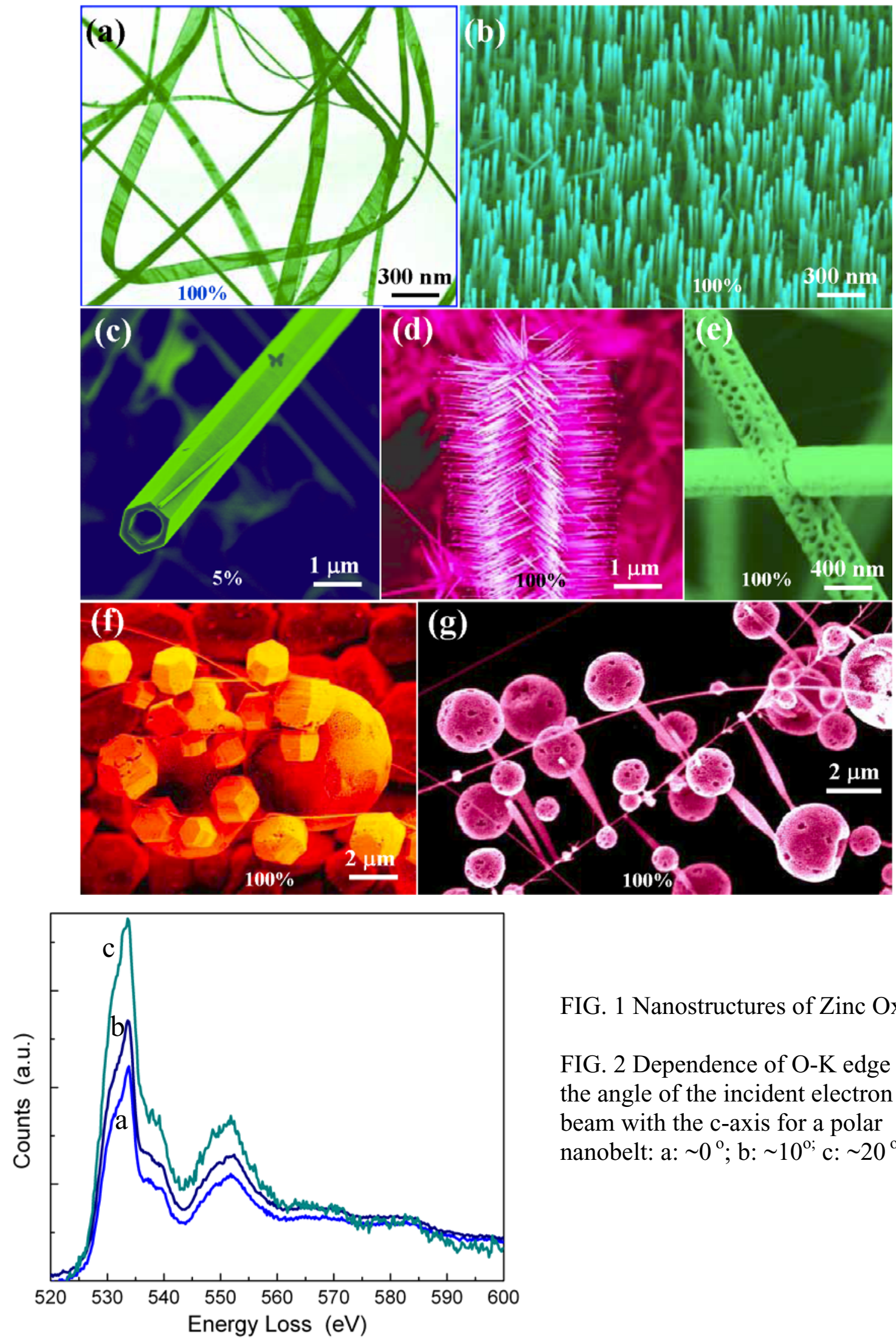

FIG. 1 Nanostructures of Zinc Oxide.

FIG. 2 Dependence of O-K edge on the angle of the incident electron beam with the c-axis for a polar nanobelt: a: $\sim 0^{\circ}$; b: $\sim 10^{\circ}$; $\mathrm{c}: \sim 20^{\circ}$. 